\title{
Enhanced Oxygen Permeation through Surface Modified Blend Films Containing Polydimethylsiloxane Graft Copolymers
}

\author{
Yuhsuke KAWAKAMI, ${ }^{*}$ Toshiki AOKI, and Yuya YAMASHITA \\ Department of Synthetic Chemistry, Faculty of Engineering, Nagoya University, \\ Chikusa, Nagoya 464, Japan
}

(Received July 1, 1985)

\begin{abstract}
Permeability and selectivity of oxygen permeation through cast polystyrene and poly(methyl methacrylate) films were considerably enhanced by the addition of small amounts of surface active polydimethylsiloxane graft copolymer. Factors controlling the permeation behavior through the surface modified films are discussed.

KEY WORDS Polydimethylsiloxane / Graft Copolymer / Blend / Film /

Surface Modification / Oxygen Permeation / Permeability / Dissolution /

Diffusion /
\end{abstract}

Polydimethylsiloxane-branched graft copolymers were found to show interesting surface active properties when added to a substrate polymer film. Examples of surface modifications of cast films of less hydrophobic substrate polymers to a hydrophobic surface by the surface accumulation of graft copolymers in the cast blend films have been reported. ${ }^{1-3}$ ESCA, FT-ATRIR, and electron microscopic studies revealed that the surface accumulated polydimethylsiloxane graft copolymers formed island domains at the surface. $^{3}$ Such polydimethylsiloxane domains might act as highly gas dissoluble surfaces and influence gas permeation behavior through the cast blend film.

In this article, we report on enhanced oxygen permeation through the surface modified cast films of polystyrene(polySt), poly(methyl methacrylate) (polyMMA), or poly(dimethylsiloxane-b-bisphenol A carbonate) (PDMS/ PC) by the addition of small amounts of surface active PDMS-polySt or -polyMMA graft copolymers.

\section{EXPERIMENTAL}

Polydimethylsiloxane-graft copolymers (GM211, GS211) were synthesized as reported earlier by the use of polydimethylsiloxane macromers. $^{2}$ PDMS/PC was prepared similarly to the literature. ${ }^{4}$ The characterization of the graft copolymers and substrate polymers are tabulated in Table I. Films of substrate polymers (polySt, polyMMA, or PDMS/PC) or their binary blends containing the graft copolymer $(0.1-6 \%$ by wt) were prepared from $5 \%$ THF solution by casting technique. The films were dried under vacuum for $24 \mathrm{~h}$, and annealed at $50^{\circ} \mathrm{C}$ for $24 \mathrm{~h}$. The thickness of the films was about $80 \mu \mathrm{m}$. The permeability and selectivity in permeation were evaluated for supplied air by the gas chromatographic method at $25 \pm 1^{\circ} \mathrm{C}$. The flow diagram is reported elsewhere. ${ }^{5}$ Typical changes in amounts of permeated gases with time are shown in Figure 1.

Permeation of a gas through a condensed film is usually treated based on Fick's and Henry's laws. 
Table I. Characteristics of polymers in this study

\begin{tabular}{lcc}
\hline \multicolumn{1}{c}{ Polymer } & $M_{w} \times 10^{-4}$ & Siloxane content $/ \mathrm{wt} \%$ \\
\hline PolySt & 31 & - \\
PolyMMA & 15 & - \\
PDMS/PC & 4.2 & 40 \\
GS211 & 5.8 & 53 \\
GM211 & 7.8 & 42 \\
\hline
\end{tabular}

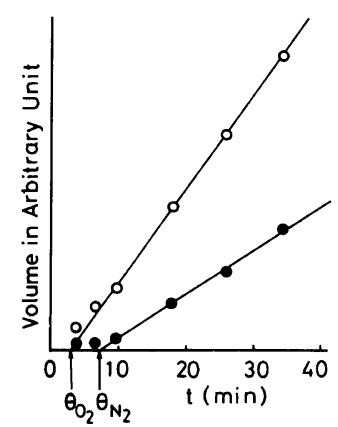

Figure 1. Typical permeation behavior of air through blend film of GS211 (2 wt \%) in polySt.

$$
q=P p_{1} A(t-\theta) / l
$$

where, $D$ and $S$ are diffusion and solubility coefficients, and $p_{1}$ is the partial pressure of the permeate at upstream side (pressure of down stream side is approximated to be 0 ), $A$ is area of permeation, $l$, thickness of the film, $t$, time, and $\theta$ is the time lag in permeation.

The permeability coefficient $P$ is defined as the product of $D$ and $S$.

$$
P=D S
$$

The diffusion coefficient $D$ can be correlated to $\theta$ by eq 3 .

$$
\begin{aligned}
& D=R^{R} / 6 \theta \\
& S=P / D
\end{aligned}
$$

By measuring $q, p_{1}, A, l$, and $t, \theta$ and $P$ were estimated by the least squares method, and $D$ and $S$ were caluculated by eq 2 and $3 .^{6-8}$ Selectivity in permeation was defined by eq 4 .

$$
\alpha=P_{\mathrm{O}_{2}} / P_{\mathrm{N}_{2}}
$$

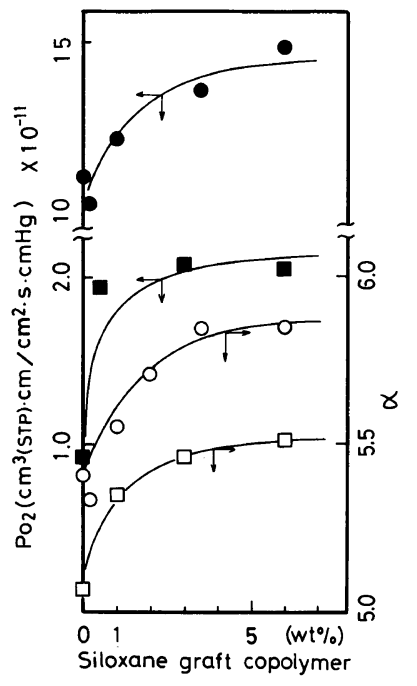

Figure 2. Change of oxygen permeability coefficient and selectivity in permeation with the concentration of added graft copolymer: permeation coefficient of the blend film of GS211 in polySt; $\bigcirc$, selectivity in the permeation; $\square$, permeation coefficient of the blend film of GM211 in polyMMA; $\square$, selectivity in the permeation.

\section{RESULTS AND DISCUSSION}

The changes of oxygen permeability coefficient and selectivity in permeation are shown in Figure 2.

When graft copolymers were added, the permeability coefficients increased with increasing concentration of the graft copolymers in the blend. With small amounts (less than $1 \%$ ) of added graft copolymer GS211, the $P_{\mathrm{O}_{2}}$ values tended to decrease momentarily. The reason is not clear at present. Barrie ${ }^{9}$ reported the differences in permeation behaviors of gaseous hydrocarbons through the films of PDMS-polySt block and graft copolymers and indicated that the different behaviors came from differences in the miscibility of the two components in the block and graft copolymers resulting in the difference in the amounts of microvoids. In the blend system of the present study, the behavior of the surface active graft copolymer is not clear 
Table II. Permeability coefficients and selectivity in permeation through PDMS/PC based blend films

\begin{tabular}{lll}
\hline Additive $/ \mathrm{wt} \%$ & $P_{\mathrm{O}_{2}}{ }^{\mathrm{a}}$ & $\alpha$ \\
\hline None & 3.56 & 2.29 \\
GS211, 1.0 & 3.29 & 2.22 \\
PolySt, 2.0 & 1.77 & 2.57
\end{tabular}

"In $10^{-9} \mathrm{~cm}^{3}$ (STP) $\mathrm{cm} \mathrm{cm}^{-2} \mathrm{~s}^{-1} \mathrm{cmHg}^{-1}$

at present, but it may change the solubility behavior of the air in the blend from that of polySt. It will be necessary to carry out more detailed experiments in order to elucidate this phenomenon. The results of the effects of the addition of polySt or GS211 into PDMS/PC are shown in Table II. When GS211 was added to PDMS/PC, the permeability coefficient decreased and selectivity in permeation also decreased slightly. With the addition of polySt, permeability coefficient decreased but selectivity in permeation increased.

In order to obtain more insight as to the permeation mechanism, the permeation behavior of such blend film was analyzed by morphology models. Results of the analyses by serial model ${ }^{10}$ are shown in Tables III and IV. Since the permeation coefficient of GS211 itself could not be determined because of its lack in film forming property, the blend GS211 in PDMS/PC is assumed to be composed of polySt and PDMS/PC in the analysis by serial model. Namely, the permeation coefficient of GS211 in the blend was substituted by that of polySt. Similarly, in the case of the blend GS211 in polySt, the permeation coefficient of GS211 is substituted by that of polydimethylsiloxane. In the case of GS211 in PDMS/PC, the calculated values are quite comparable with the expected values from the amounts of added graft copolymers, irrespective of the direction of the permeation from the air side surface or glass side surface, a fact which suggests the compatible nature of the two polymers. When air was permeated from the air side surface of the cast film of polySt
Table III. Analysis of the permeation behavior of polySt based blend film containing GS211 by serial model"

\begin{tabular}{ccc}
\hline $\begin{array}{c}\text { Content of dimethylsiloxane } \\
\text { unit in the blend } / \mathrm{wt} \%\end{array}$ & $P_{\mathrm{O}_{2}}{ }^{\mathrm{b}}$ & $L / \%$ \\
\hline None & 1.17 & - \\
0.53 & 1.22 & 4.11 \\
1.86 & 1.36 & 14.0 \\
1.86 & $1.20^{\mathrm{c}}$ & 2.51 \\
3.18 & 1.49 & 21.5 \\
3.18 & $1.30^{\mathrm{c}}$ & 15.9 \\
\hline
\end{tabular}

a $P_{1}=1.17 \times 10^{-10} \mathrm{~cm}^{3}(\mathrm{STP}) \mathrm{cm} \mathrm{cm}^{-2} \mathrm{~s}^{-1} \mathrm{cmHg}^{-1}$. $P_{2}=3.52 \times 10^{-8} \mathrm{~cm}^{3}$ (STP) $\mathrm{cm} \mathrm{cm}^{-2} \mathrm{~s}^{-1} \mathrm{~cm} \mathrm{Hg}^{-1} \cdot{ }^{10}$

b In $10^{-10} \mathrm{~cm}^{3}$ (STP) $\mathrm{cm} \mathrm{cm}^{-2} \mathrm{~s}^{-1} \mathrm{cmHg}^{-1}$.

c Permeation from glass side surface.

Table IV. Analysis of the permeation behavior through the PDMS/PC based blend films by serial model ${ }^{a}$

\begin{tabular}{rllc}
\hline Additive (wt $\%$ of the PolySt unit) & $P_{\mathrm{O}_{2}{ }^{\mathrm{b}}}$ & $L / \%$ \\
\hline None & & 3.56 & - \\
GS211 & 0.47 & 3.27 & 0.29 \\
PolySt & 2.0 & 1.77 & 3.56 \\
\hline
\end{tabular}

$P_{1}=3.56 \times 10^{-9} \mathrm{~cm}^{3}(\mathrm{STP}) \mathrm{cm} \mathrm{cm}^{-2} \mathrm{~s}^{-1} \mathrm{cmHg}^{-1}$.
$P_{2}=1.17 \times 10^{-10} \mathrm{~cm}^{3}(\mathrm{STP}) \mathrm{cm} \mathrm{cm}^{-2} \mathrm{~s}^{-1} \mathrm{cmHg}^{-1}$.
b In $10^{-9} \mathrm{~cm}^{3}(\mathrm{STP}) \mathrm{cm} \mathrm{cm}^{-2} \mathrm{~s}^{-1} \mathrm{~cm} \mathrm{Hg}^{-1}$.

containing small amounts of GS211, where the polydimethylsiloxane graft copolymers have accumulated, ${ }^{3}$ the calculated values of $L$ were much larger than the expected values from the added graft copolymers. When air was permeated from the glass side surface of the film, where the graft copolymers have accumulated but to lesser extent than from the air side surface, ${ }^{3}$ the value of $L$ became smaller but still quite larger than the expected values. This may indicate the importance of the solubility of gases at the surface of the blend films. Here, a brief comment on the appropriatness of such treatment will be made. The $L$ in the serial model can be expressed as eq 5.

$$
L=\left(P-P_{1}\right) P_{2} /\left(P_{2}-P_{1}\right) P
$$

In case of GS211 (3.50 wt $\%$ ) in polySt, which 
corresponds to a blend film containing $1.86 \mathrm{wt} \%$ polydimethylsiloxane unit in polySt, $P_{2}$ was taken as $3.52 \times 10^{-8} \mathrm{~cm}^{3}(\mathrm{STP}) \mathrm{cm}$ $\mathrm{cm}^{-2} \mathrm{~s}^{-1} \mathrm{cmHg}^{-1}$. Since $P$ and $P_{1}$ are 1.36 and 1.17 in $10^{-10} \mathrm{~cm}^{3}(\mathrm{STP}) \mathrm{cm} \mathrm{cm}^{-2} \mathrm{~s}^{-1}$ $\mathrm{cmHg}^{-1}$ unit, respectively, the value of $L$ should remain almost constant even if $P_{2}$ (permeability coefficient of the added graft copolymer) decreased to 11.7 in $10^{-10}$ unit (10 times of $\left.P_{1}\right)$, since $L$ can be approximated as $L=\left(P-P_{1}\right) / P$ under the condition of $P_{2} \gg P_{1}$. In case of GS211 $(1.0 \mathrm{wt} \%)$ in PDMS/PC, which corresponds to a blend film containing $0.47 \mathrm{wt} \%$ polySt in PDMS/PC, $P_{2}$ was taken as $1.17 \times 10^{-10} \quad \mathrm{~cm}^{3}(\mathrm{STP}) \mathrm{cm} \quad \mathrm{cm}^{-2} \mathrm{~s}^{-1}$ $\mathrm{cmHg}^{-1}$, and since $P$ and $P_{1}$ are 3.27 and 3.56 in $10^{-9} \mathrm{~cm}^{3}$ (STP) $\mathrm{cm} \mathrm{cm}^{-2} \mathrm{~s}^{-1} \mathrm{cmHg}^{-1}$ unit, respectively, $L$ would become $4.3 \%$ if $P_{2}$ increases to 1.17 in the $10^{-9}$ unit, which is considerably larger than the expected value from the amounts of added graft copolymer. This might indicate the importance of the phase separated structure of the blend films. However, this does not seem to be the case because permeation coefficients from both the air and glass side surfaces were the same as mentioned earlier. Similar results were obtained in the GM211-polyMMA system.

In order to clarify the importance of the solubility factor in the permeation, the permeability coefficients were separated into diffusion and solubility coefficients, and the results are shown in Figure 3.

In polySt-GS211 blend films, the diffusion coefficients increased only a little with increasing concentration of added graft copolymer. The solubility coefficient increased very sharply with the concentration of the graft copolymers (up to $2 \%$ ). These changes are quite similar to the changes in $P_{\mathrm{O}_{2}}$ and $\alpha$. As can be seen from Figure 3, the reason for the higher selectivity of oxygen permeation over nitrogen through the blend film, compared with the substrate polymer film, seems to lie in the relatively higher solubility coefficient of oxygen than nitrogen at the surface. Such

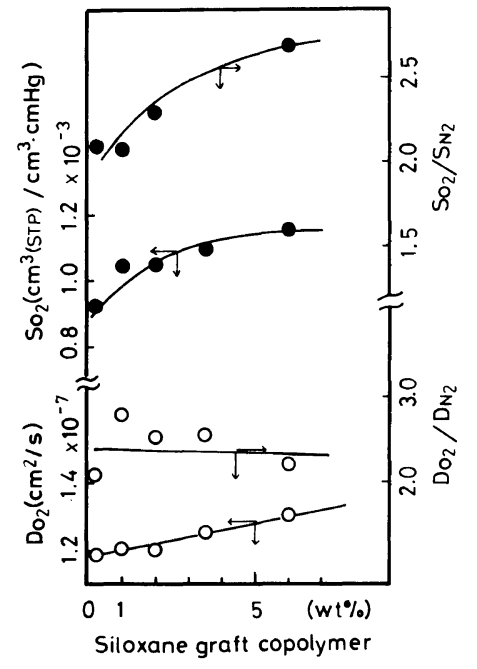

Figure 3. Change in solubility and diffusion coefficients of polySt with the concentration of added GS211.

Table V. Solubility coefficients of polySt and PDMS

\begin{tabular}{ccc}
\hline Polymer & $S_{\mathrm{O}_{2}{ }^{2}}{ }^{a}$ & $S_{\mathrm{O}_{2}} / S_{\mathrm{N}_{2}}$ \\
\hline PolySt $^{12}$ & 2.2 & 1.9 \\
PDMS $^{13}$ & 4.1 & 2.2 \\
\hline
\end{tabular}

a In $10^{-3} \mathrm{~cm}^{3}$ (STP) $\mathrm{cm}^{-3} \mathrm{cmHg}^{-1}$.

change in permeation behavior might reflect the actual morphology of the film surface. As already pointed out, the polydimethylsiloxane graft copolymers accumulate at the film surface, and may play an important role in changing the concentration of gases at the surface. If such domains at the surface have relatively higher solubility of oxygen over nitrogen, the selectivity in permeation will also increase with the increase in permeability. The solubility coefficients of oxygen and nitrogen into PolySt and PDMS are shown in Table V. ${ }^{11,12}$

As shown in Table $\mathrm{V}$, polydimethylsiloxane is considered to have relatively higher solubility of oxygen over nitrogen than polySt or polyMMA. ${ }^{12,13}$ Relatively higher increase in solubility of oxygen over nitrogen into the 
polydimethylsiloxane modified surface seems to be the reason for higher permeability and selectivity of oxygen over nitrogen through the blend film. In the case of polyMMA blend film with GM211, the trend was similar to polySt blend film although the change was smaller.

If a very simplified assumption is made that $A$ (polyDMS area) controls the concentrations of gases, the permeation behavior can be discussed based on the changes in $A$ and $d$. The changes of $A$ and $d$ by composite model (Figure 4$)^{14}$ are shown in Figure 5. Although it is very difficult to discuss quantitatively, the trend may be discussed qualitatively. When small amounts of graft copolymer $(<1 \%)$ were added, the value $A$ sharply increased, which may indicate that the copolymers locate as small spheric domains at the surface. With increasing concentration, $A$ increases sharply with moderate increase in $d$, a fact which may suggest the spreading of the PDMS domain at the surface. Thus, the solubility of gases is considered to increase, resulting in the increase in permeability coefficient. Such increase in $A$ levelled off at a concentration of about $1 \%$ with $d$ still increasing. The change in permeation is consistent with the change in contact angle, which is a measure of hydrophobicity of the surface by surface modification, also shown in the same Figure. This fact seems to indicate the importance of the PDMS domain at the surface to determine permeability. At higher concentrations, the morphology of the film may change and cannot be discussed on the same bases. Thus, the role of the surface of the phase-separated domain is shown to be specially important to control the permeation when the concentration of added graft copolymer is low. In case of PDMS/PC with GS211, the compatibility of the added graft copolymer with substrate polymer is considered to be much better than the combination of polySt--GS211, and the film could be analyzed as a serial model as already shown in

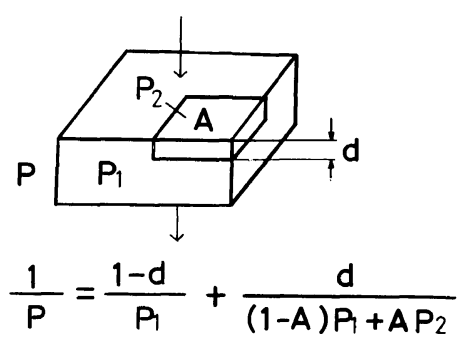

Figure 4. Composite model in permeation.

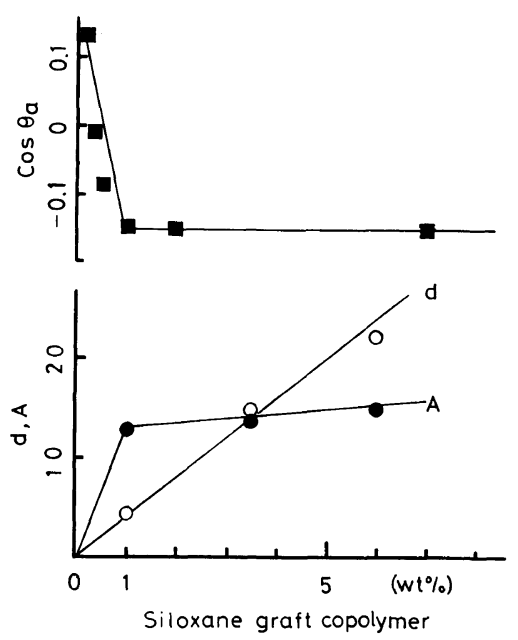

Figure 5. Analysis of permeation behavior the blend film of GS211 in polySt by composite model."

"Contact angle changes were measured on the blend film of GM211 in polyMMA. ${ }^{1}$

\section{Table III.}

As discussed above, this work opens up a new possibility to controll permeation behavior by surface morphology through surface accumulation of graft copolymer in the blend film. Further research is needed to clarify the precise mechanism.

Acknowledgement. Partial financial support by Kurata Foundation, Ishida Foundation, and a Grant-in-Aid for Scientific Research (No. 60550639) by Ministry of Education, Science and Culture are gratefully acknowledged. 


\section{REFERENCES}

1. Y. Kawakami, R. A. N. Murthy, and Y. Yamashita, Polym. Bull., 10, 368 (1983).

2. Y. Yawakami, R. A. N. Murthy, and Y. Yamashita, Makromol. Chem., 185, 9 (1984).

3. Y. Kawakami, T. Aoki, and Y. Yamashita, Macromolecules, 18, 580 (1985).

4. H. A. Vaughn, J. Polym. Sci., B, 7, 569 (1969).

5. Y. Kawakami, H. Karasawa, T. Aoki, Y. Yamamura, H. Hisada, and Y. Yamashita, Polym. J., 17, 1159 (1985).

6. H. A. Daynes, Proc. R. Soc. London, Ser. A, 97, 286 (1920).

7. R. M. Barrer, Trans. Faraday Soc., 35, 628, 644
(1939).

8. W. A. Rogers, R. S. Burity, and D. Alpert, J. Appl. Phys., 25, 868 (1954).

9. J. A. Barrie and K. Munday, J. Membrane Sci., 13, 175 (1983).

10. J. E. Nielson, "Mechanical Properties of Polymers and Composites" Marcel Dekker, New York, N. Y., 1974.

11. T. Nakagawa, "Makugaku Nyuumon," Kitami Syobou, Tokyo, 1978, p 273.

12. Y. Kawakami, H. Karasawa, H. Kamiya, T. Aoki, and Y. Yamashita, Polym. J., 18, 237 (1986).

13. W. L. Robb, Anal. N. Y. Acad., 146, 119 (1967).

14. M. Takayanagi, S. Uemura, and S. Minami, $J$. Polym. Sci., C, 5, 113 (1964). 